\title{
COVID-19 Experience in Hemodialysis Patients: A Cue for Therapeutic Heparin-Based Strategies?
}

\author{
Antonio Pisani ${ }^{a}$ Manuela Rizzo ${ }^{a}$ Valentina Angeluccia Eleonora Riccio ${ }^{b}$ \\ aDepartment of Public Health, Chair of Nephrology, University Federico II of Naples, Naples, Italy; ${ }^{b}$ Institute for \\ Biomedical Research and Innovation, National Research Council of Italy, Palermo, Italy
}

\section{Keywords}

Coronavirus disease $2019 \cdot$ Hemodialysis · Heparin

\begin{abstract}
In our opinion, the use of heparin could play a crucial role in these patients. In fact, recent studies have shown that heparin, the most commonly used anticoagulant during HD procedures, had anti-inflammatory properties and a direct antiviral action, due to its ability to prevent SARS-CoV-2 pseudovirus entry into host cells. These activities, together with its anticoagulant action, could explain the ability of heparin to ameliorate COVID-19 clinical course.

๑) 2020 S. Karger AG, Basel
\end{abstract}

\section{Introduction}

Coronavirus disease 2019 (COVID-19) is an outbreak due to SARS-CoV-2, a new virus of Coronaviridae family, emerged in China in December 2019 and declared by the World Health Organization a global pandemic on March 2020. The infection causes an atypical flu that often leads to severe acute respiratory syndrome with high mortality

karger@karger.com

(c) 2020 S. Karger AG, Basel

www.karger.com/nef

Karger rate. Patients with underlying diseases, such as those suffering from ESRD, are at greater risk.

In particular, COVID-19 is a real challenge for patients on hemodialysis (HD), whose high susceptibility to COVID-19 is only partially explained by their average old age, frequent comorbidities, and impaired immune function. In fact, the need of physical presence at health care facilities, the mobility to HD centers, and the high aggregation for the continuous physical proximity of patients during HD session strongly increase the risk of infection [1]. Surprisingly, first available data suggest that although HD patients' infection rate in the COVID-19 epidemic is much higher than in other populations, the disease course is rarely fatal and less severe, both compared with kidneytransplanted patients and general population [1].

Most of current knowledge on COVID-19 impact on HD patients derives from a report of a single HD center in Renmin Hospital in Wuhan, Hubei, China [2]: from January 14, 2020, the day of confirmation of the first case, to February 17, 2020, the day of the end of epidemic, there were a total of 37 cases (16\%) of COVID-19 among 230 HD patients. In most patients, the COVID-19 symptoms were mild ( $11 \%$ of patients had fever, $8 \%$ fatigue, and only $3 \%$ cough, chest pain, or nausea), and no patient was ad- 


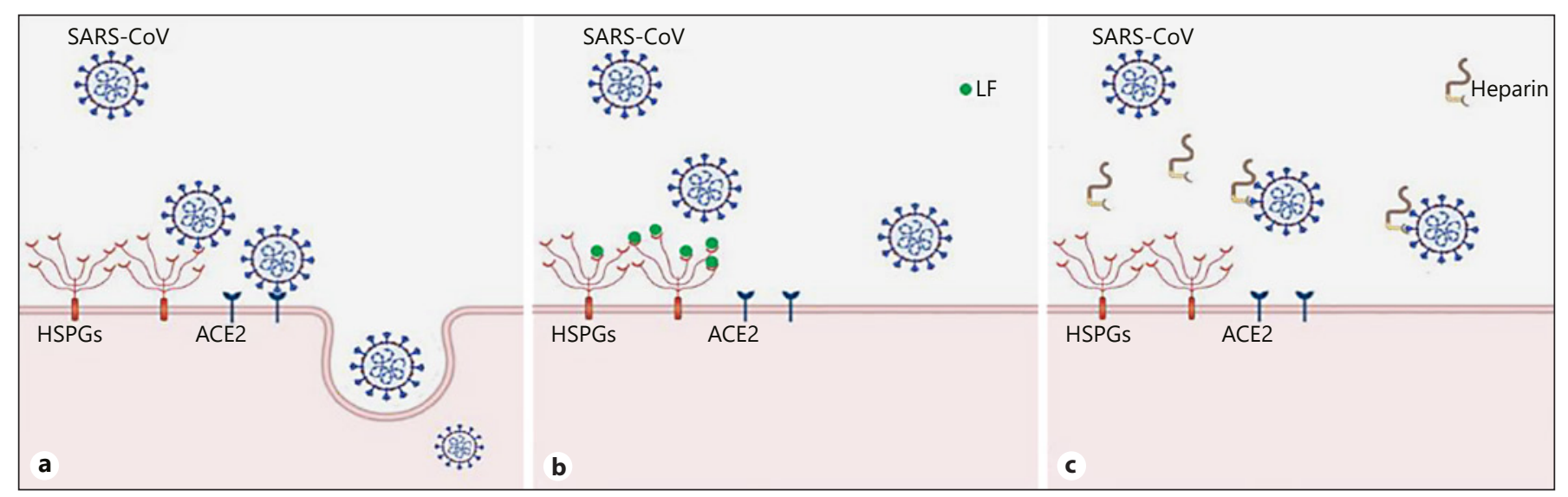

Fig. 1.SARS-COV-2 entry in host cells and hypothetical protective role of lactoferrin and heparin. a SARS-COV-2 cell entry: The anchorage provided by the HSPGs allows initial contact between SARS-CoV-2 and host cells on the cell surface. SARS-CoV-2 then rolls onto the cell membrane by binding to specific input receptors, ACE2, which leads to subsequent cell entry. b Protective role of lactoferrin: LF blocks the interaction between SARS-CoV-2 and HSPGs. c Protective role of heparin: Heparin prevents the entry of SARS-CoV-2 into cells, binding to the recombinant surface receptor-binding domain (SARS-CoV-2 S1 RBD) and inducing a significant structural change. HSPG, heparan sulfate proteoglycan; ACE2, angiotensin-converting enzyme 2; LF, lactoferrin. mitted to intensive care units. Six HD COVID-19 patients died, but the causes of death were apparently not related to pneumonia. Interestingly, infected HD patients showed a remarkable reduction in the numbers of $\mathrm{T}$ cells, $\mathrm{T}$ helper cells, killer T cells, and NK cells in peripheral blood mononuclear cells (PBMCs), as well as lower serum levels of inflammatory cytokines, compared to no-HD COVID-19 patients. More recent data, collected in the Italian region with the major SARS-CoV-2 spread [3] and in Zhongnan Hospital of Wuhan University [4], seem to support Chinese conclusions about less severe clinical course in HD population.

\section{Why COVID-19 May Be Less Severe in Dialysis Patients?}

The reduced function of the immune system and decreased cytokine storm reported in Wuhan HD population only partially explain this phenomenon [2]. In fact, it has been shown that the mechanism leading to severe condition or death in COVID-19 patients is the overresponse against the virus by the immune system with the cytokine storm [5].

However, interesting cues may emerge from scientific literature regarding 2002-2004 SARS outbreak, caused by SARS-CoV, a different but related virus in the Coronaviridae family. A 2011 paper investigated SARS-CoV mechanisms of adhesion and entry into host cells [6].
SARS-CoV spike protein is the envelope protein responsible for invasion of host cells, through the binding to specific receptors. The heparan sulfate proteoglycan (HSPG), ubiquitary cell-surface molecules, and particularly heparan sulfate (HS) chains, play a crucial role in this process, providing the first anchoring site on the cell surface. The anchorage provided by the HSPGs allows initial contact between SARS-CoV and host cells on the cell surface. SARS-CoV then rolls onto the cell membrane by binding to specific input receptors, as angiotensin-converting enzyme 2, a metallopeptidase identified as one of its functional receptors, which leads to subsequent cell entry (Fig. 1a).

In this scenario, lactoferrin (LF), a multifunctional protein present in external secretions and an important constituent of the neutrophilic granules of leukocytes, has been shown to possess strong antiviral activity against a broad spectrum of viruses, by binding to HSPGs and preventing the preliminary interaction between the virus and host cells [6] (Fig. 1b). Similarly, heparin, a highly sulfated glycosaminoglycan widely used as anticoagulant, has been shown to prevent SARS-CoV [6] and, recently, SARS-CoV-2 [7] pseudovirus entry into host cells. Specifically, the polydisperse, natural products of HS and the soluble HS analog polysaccharide heparin have been found to be involved and prevent infection by a range of viruses, including SARS-CoV-2. A recent experiment demonstrated an interaction between the recombinant surface receptor-binding domain (SARS-CoV-2 S1 RBD) 
and heparin, resulting in a significant structural change and avoiding the SARS-CoV-2 entry into host cells [7] (Fig. 1c).

Thus, heparin protection against SARS-CoV-2 may offer further explanation for benign clinical outcome in HD population. In fact, anticoagulation is essential to $\mathrm{HD}$, and heparin is the most commonly used anticoagulant during HD procedures. In addition, the use of anticoagulant therapy with heparin in severe COVID-19 patients [8], and in particular, in those meeting sepsis-induced coagulopathy criteria or with markedly elevated D-dimer $[9,10]$ has been associated with a better prognosis. In fact, acute respiratory distress syndrome is one of the commonest complications of COVID-19 infection, and activation of the coagulation system has been shown to be relevant in its pathogenesis.

However, it is not to be excluded that the beneficial effect of heparin on the clinical course of COVID-19 could be partially explained with its non-anticoagulant actions. In fact, one of the better known non-anticoagulant properties of heparin, its anti-inflammatory function, may also be relevant in this setting $[11,12]$, as well as the direct antiviral action.

Taken together, these observations, although are all based only on indirect evidences, seem to support the hypothesis of a crucial role of use of heparin in the less severe clinical course of COVID-19 in HD patients. Further studies in larger heparin-treated populations are needed to confirm the role of different types of heparin as a countermeasure against the current SARS-CoV-2 outbreak.

\section{Disclosure Statement}

The authors have no conflict of interest to declare.

\section{Author Contributions}

Antonio Pisani had the original idea; Emanuela Rizzo, Valentina Angelucci, and Eleonora Riccios studied the literature; and Antonio Pisani and Eleonora Riccio wrote the paper. All authors read and approved the final version.

\section{References}

1 Ikizler TA. COVID-19 and dialysis units: what do we know now and what should we do? Am J Kidney Dis. 2020 Mar 23;S02726386(20):30608-9.

2 Ma Y, Diao B, Lv X, Zhu J, Liang W, Liu L, et al. 2019 novel coronavirus disease in hemodialysis (HD) patients: report from one HD center in Wuhan, China. medRxiv. 2020 Feb; 2020. https://dx.doi.org/10.1101/2020.02.24. 20027201.

3 Alberici F, Del Barba E, Manenti C, Econimo L, Valerio F, Pola A, et al. "Brescia renal covid task force". Managing patient in dialysis and kidney transplant infected with Covid-19. G Ita Nefrol. 2020;37(2):2020-vol2.

4 Wuang R, Liao C, He H, Hu C, Wei Z, Hong Z, et al. COVID-19 in hemodialysis patients: a report of 5 cases. Am J Kidney Dis. 2020. https://dx.doi.org/10.1053/j.ajkd.2020. 03.009 .
5 Huang C, Wang Y, Li X, Ren L, Zhao J, Hu Y, et al. Clinical features of patients infected with 2019 novel coronavirus in Wuhan, China. Lancet. 2020;395(10223):497-506.

6 Lang J, Yang N, Deng J, Liu K, Yang P, Zhang $\mathrm{G}$, et al. Inhibition of SARS pseudovirus cell entry by lactoferrin binding to heparan sulfate proteoglycans. PLoS One. 2011;6(8):e23710. Epub 2011 Aug 22.

7 Mycroft-West C, Su D, Elli S, Guimond S, Miller G, Turnbull J, et al. The 2019 coronavirus (SARS-CoV-2) surface protein (Spike) S1 Receptor Binding Domain undergoes conformational change upon heparin binding. BioRxiv. Preprint.

8 Shanghai Clinical Treatment Expert Group for COVID-19. Comprehensive treatment and management of coronavirus disease 2019: expert consensus statement from Shanghai (in Chinese). Chin J Infect. 2020;38. Published online ahead of print. BioRxiv. Preprint. https://doi.org/10.1101/2020.02.29. 971093.
9 Tang N, Bai H, Chen X, Gong J, Li D, Sun Z Anticoagulant treatment is associated with decreased mortality in severe coronavirus disease 2019 patients with coagulopathy. J Thromb Haemost. 2020;18:1094-9. http:// dx.doi.org/10.1111/jth.14851.

10 Thachil J. The versatil heparin in COVID-19. J Thromb Haemost. 2020;18:1020-2. http:// dx.doi.org/10.1111/jth.14879.

11 Poterucha TJ, Libby P, Goldhaber SZ. More than an anticoagulant: do heparins have direct anti-inflammatory effects? Thromb Haemost. 2017 Feb 28;117(3):437-44.

12 Mousavi S, Moradi M, Khorshidahmad T, Motamedi M. Anti-inflammatory effects of heparin and its derivatives: a systematic review. Adv Pharmacol Sci. 2015;2015:507151. 